\title{
A Study on System Innovation Theory Applied to Improve the Multilanguage Guiding Device
}

\author{
Ching-Yun Huang, a, Ying-Hsiang Lin $^{2, b}$, \\ Chiapao Chang ${ }^{3, c^{*}}$ and Chien-Yu Lu ${ }^{4, d}$ \\ ${ }^{1,3}$ National Chin-Yi University of Technology, Taichung, Taiwan, R.O.C. \\ ${ }^{2,4}$ Department of Industrial Education and Technology, National Changhua University of Education, \\ Changhua, Taiwan, R.O.C \\ aab402cc@gmail.com, b3150316@yahoo.com.tw, chiapc@ncut.edu.tw, \\ lab402bb@gmail.com \\ ${ }^{*}$ The Corresponding author
}

Keywords: Multilanguage guiding device; TRIZ; Contradiction matrix

\begin{abstract}
A convenient portable Multilanguage guiding device is provided to promote the competitiveness of museums and the economic development of relevant industries. This study applies TRIZ innovation theory with its contradiction matrix and 40 inventive principles to the innovation and improvement of the device. The improved device provide detailed introduction of museums and guiding service for visitors from different countries. With the device, visitors could enjoy a more unrestrained sightseeing and museums would pay a lower personnel expenses for docents.
\end{abstract}

\section{Introduction}

Museums encourage innovation and regeneration of traditional culture, wider uses of collection resources, and more economic benefits. With the promotion of competitiveness, museums could also help develop the relevant industries and economy. Life-oriented education would be enhanced and popularized, recreational function would be served, and image of the city and country would be promoted altogether (Huang et. al., 2014). But for the introduction of museums, paper reading material provides limited information. Visitors' comprehension and languages would be different and need translation machines for correct information. Museums could also be introduced by docents. Problems are that visitors need to follow the regulated route for guiding. They could not enjoy the sightseeing according to their personal preferences. One docent could not be all-rounder and command so many languages for people from different countries.

The innovative (inventive) problem-solving theory TRIZ (Song, 2009) was proposed by the former Soviet Union inventor G. S. Altshuller in 1946. He was respectfully addressed as the Father of TRIZ, leading research institutions, academies, universities, and businesses. They organized themselves into the TRIZ research team. Basic theory about invention problems based on dialectical materialism with system theory was put forward after the analysis of 2,500,000 high-level patented inventions. The theoretical core includes the basics and principles, specifically includes:

1. Introduction (Basic rules, Contradiction analysis, Invention rating)

2. Technological Evolution

3. 39 Problem-solving Technical Parameters and 40 Inventive Methods

4. Object Field Analysis with Transformation Theory and 76 Standard Problem-Solving Approaches

5. Procedures to solve invention problems

6. Physical Effect Database

At present, a number of scholars are carrying out research into TRIZ field, such as Chang and Lin (2014), Chang et al. (2015) and Lin et al. (2016). TRIZ theory of contradiction matrix, 39 technical parameters and 40 invention innovation methods were used to identify and solve problems 
and provide improvements.

\section{Methodology}

Research Methodical Procedures. After identifying a problem, we find the problem point and technical contradictions. With the contradictions, we drew the $39 \times 39$ matrix, looked for possible problem-solving methods corresponding to the contradictions, listed the solutions with logical thinking, and then found out the most appropriate programs and solved the problems finally.

Contradiction Matrix. Altshuller observed every technical contradiction, developed a system corresponding to invention and innovation, and proposed the first contradiction matrix. Whenever studying a patent, he tried to classify and confirm the contradictions and applied principles corresponding to its innovative invention. Results he induced from large number of patents were summarized into 39 technical parameters, 40 inventive principles and formulated the $39 \times 39$ matrix (Table 1) represented and structured by $\mathrm{i}$ for improved characteristics and $\mathrm{j}$ for avoidance of worsening results. And each grid represented one type of invention problems respectively. Each problem had its own corresponding $\mathrm{i}$ and $\mathrm{j}$ coordinates. In the specific $\mathrm{i}$ and $\mathrm{j}$ grids, all problem-solving inventive principles had their own identification codes (Chiang, 2008).

40 Inventive Principles. 40 Principles provide the TRIZ users with a thought process from theory to practice. Every invention theory is a simple fundamental concept, but its derivative thoughts could comprehend a very wide range of study fields (Pan \& Hsiao, 2007)

\section{Improved Design of the Multilanguage Guiding Device}

Technical Contradictions of the Multilanguage Guiding Device. Museums provide guiding service for people from different countries through paper reading material or books, but the information provided is limited. Besides, visitors' comprehension would be different. If detailed introduction is needed, enough collection of contents and selection of languages must be provided. It will cause the waste of resources. Docents couldn't be all-rounders and couldn't command every language of different countries. If visitors ask for sightseeing according to their own preferences, more docents must be needed and this surely will increase the burden of museums.

Technical contradictions of the Multilanguage device lie in: the required "speed" catering to language needs of visitors from different countries and the "material loss" caused by the need of multilingual comprehensive information, hence by the need of more paper or book reading guide, hence by the need for more docents.

Draw the Contradiction Matrix. As shown in Table 1, find out the appropriate i (improved characteristics) and $\mathrm{j}$ (avoidance of worsening results) from the matrix. "Speed" in Row 9 corresponds to "material loss" in Column 23.The intersection grids represent the suggested problem-solving principles for the contradictions.

Table 1 Contradiction matrix

\begin{tabular}{|c|c|c|c|c|}
\hline \multirow{2}{*}{\multicolumn{2}{|c|}{$\begin{array}{l}\text { The avoidance of } \\
\text { worsening result }(\mathrm{j})\end{array}$}} & 1 & 23 & 39 \\
\hline & & $\begin{array}{l}\text { Weight of } \\
\text { the moving } \\
\text { object }\end{array}$ & $\begin{array}{l}\text { Loss of } \\
\text { substance }\end{array}$ & $\begin{array}{l}\text { producti } \\
\text { vity }\end{array}$ \\
\hline & $\begin{array}{l}\text { Weight of the } \\
\text { moving object }\end{array}$ & & & \\
\hline \multirow{2}{*}{9} & Speed & & $10,13,28,38$ & \\
\hline & Productivity & & & \\
\hline
\end{tabular}


Found from the 40 inventive principles in the matrix, Principle 10 "Pre-function", Principle

13 "Contrary", Principle 28 "Replacement System", and Principle 38 "Use of Strong Oxidant" are state-of-the-art suggestions.

Principle 10 "Pre-function":

a. Fulfill the functions (wholly or partially) required in advance.

b. Arrange things in advance in the right and convenient places for them to perform the required functions.

By application of this principle, language types and guiding content were prerecorded in the speech recognition Multilanguage guiding device.

Principle 13 "Contrary":

a. Fulfill the contrary functions instead of the regulated functions of the subject conditions.

b. Make things or the exterior media immobile; make the immobile movable.

c. Reverse things.

By application of this principle, the Multilanguage guiding device would be convenient for visitors to carry around.

Principle 28 "Replacement System":

a. Use another sensing method.

b. Use electrical field, magnetic field or electromagnetic field and make them interact with things and systems.

c. Replace the static field with the dynamic one; replace the unstructured field with the structured one.

d. Use the field and associate the particles, things and systems that can be applied and reacting with the field.

By application of this principle, the Multilanguage guiding device can provide service to visitors anytime anywhere for any exhibition or products.

Principle 38 "Use of Strong Oxidant":

a. Replace common air with the oxygen-rich air.

b. Replace oxygen-rich air with oxygen.

c. Apply electric dissociation to air and oxygen.

d. Use ozonized oxygen

e. Replace ozonized oxygen with ozone.

By application of this principle, the Multilanguage guiding device help reduce the burden of human resources for museums.

\section{Consideration and Improvement}

According to Principle 10"Pre-function", the first thing to consider in this research is that museum visitors are from different countries and need different languages for guiding service. Hence, the device is designed to prepare for visitors a multilingual selection of introduction. Then, in consideration of Principle 13 "Contrary", it is found that museums exhibit diverse work but not exactly each piece of exhibition would attract each visitor. Visitors might have their own expected itineraries. Therefore, the device, designed for these visitors, replaces the immovable with the movable and would be easy to carry around. Moreover, Principle 28 "Replacement System" leads the consideration that the museum exhibition is a large collection of things shown to the public in different locations. Different and appropriate Multilanguage introduction would for sure be needed. Finally, the device investigated and improved through Principle 38 "Use of Strong Oxidant", would help museums reduce the personnel expenditure for docents. The Multilanguage guiding device is shown in Fig. 1. 


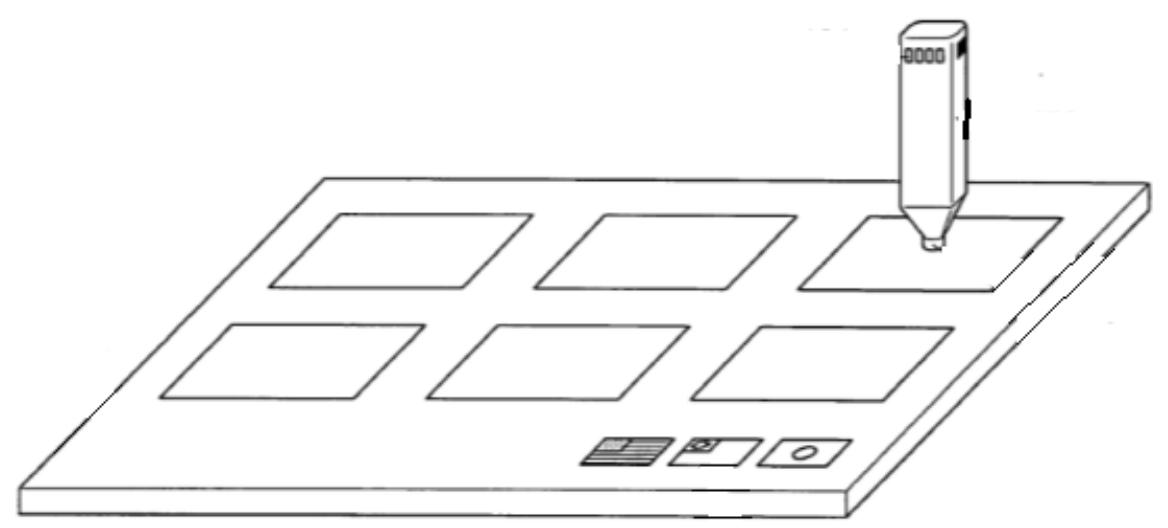

Figure 1. Finite Multilanguage guiding device

\section{Conclusion}

Nowadays, museums are trying their best to make constant efficient use of their available resources to increase their economic benefits and sharpen their competitive edge. With TRIZ innovation theory as the theoretical and inventive basis, the Multilanguage guiding device could provide comprehensive introduction for any exhibition in any places. Visitors could enjoy their sightseeing according to their own preferences. The device would also provide Multilanguage selections for guiding service; hence it would increase the visitors' satisfaction and decrease museums' personnel expenditure at the same time.

TRIZ innovation theory could be applied not only to one product but to lots of things. No matter what careers people have, problems happen. Learning TRIZ is just like opening a new window. Peoples' field of vision would be expanded. It helps us quickly find the problems, analyze the problems, and work out appropriate solutions. This study gains from TRIZ design procedures and logic concepts and hence raises the feasibility of problem-solving in design improvement.

Contributions of TRIZ to the study:

1. It could be understood form the literature review that TRIZ is a logical, systematic, and user-friendly theory. It is easy for researchers who are not experts to use or understand.

2. The $39 \times 39$ contradiction matrix and the 40 inventive principles applied in this study are a breakthrough for problem-solving. It helps researchers clearly understand the process of trouble-shooting.

3. In improving the case, it provides ways of logical thinking which could be used for design improvement.

4. Through the research refinement and innovation concepts, practical functions of the Multilanguage guiding device have been promoted favorably.

\section{Reference}

[1] Song, M. H. (2009) TRIZ System Innovation Theory and Practice. Taipei: Dingmao Books

[2] Chiang, T. L. (2008) TRIZ Invention Problem-Solving Theory. Taipei: Yuher Cultures.

[3] Pan, C. W., \& Hsiao, Y. C. (2007) TRIZ Innovative Contradiction Breakthrough. Taipei: Chiensu Workhouse.

[4] Huang, Mei-Hsien, Hung Chien-Tsai, Cheng, Yu-Ping, Lin, Mu-Ting, \& Yang, Shen-Chu. The exploration of the convergence of national archives with cultural and creative industries in UK, USA and Taiwan. File Quarterly, 13(4), (2014), pp 4-21.

[5] Lin,Y.H., Li, S.P.,Lu, C.Y., Chung, R.G., Chen, D.F. " Research into the Improvement on Hexagonal Socket by Applying the System Innovation Theory" Advances in Engineering Research, Vols. 52, (2016), pp 55-57. 
[6] Chang, C. P., Lin, Y.H. "Applying TRIZ to Improve a Guide Post", Applied Mechanics and Materials, Vols. 464 , (2014), pp 431-435.

[7] Chang, C. P., Lin, Y.H. and Lu, C. Y., "Applying TRIZ Systematic Innovation Method to Improve Urinals", Advances in Engineering Research, Vols. 22, (2015), pp 793-795. 